\title{
RAPID INDOOR DATA ACQUISITION FOR LADM-BASED 3D CADASTRE MODEL
}

\author{
A. Jamali , P. Boguslawski, E.E. Duncan, C.M. Gold and A. Abdul Rahman \\ 3D GIS Research Lab, Faculty of Geoinformation and Real Estate, Universiti \\ Teknologi Malaysia (UTM), Malaysia \\ ali.jamali.65@gmail.com, \\ pboguslawski@gmail.com, edwduncan@yahoo.com \\ chris.gold@gmail.com, alias@utm.my
}

KEY WORDS: Indoor Surveying, 3D data models, LADM, Cadastre

\begin{abstract}
:
2D cadastre has been in existence for decades and most countries have found it convenient. However, in complex situations it has been found to be inadequate as ownership becomes difficult to realise. 3D cadastre data was previously not being collected, thus, making such data even more difficult to realise. 3D data collection for 3D cadastre is one of the main issues for practising surveyors. Several ways of obtaining 3D data exist namely, traditional surveying, terrestrial laser scanning and from Computer Aided Design (CAD) sources. Various data sources have different data structure and a generalized data structure for 3D cadastre hardly reported. A simple and rapid method for indoor data acquisition is proposed. This seeks to determine if the dual half edge data structure is suitable for 3D cadastre. The dual half edge data structure is applied within a graphical user interface. The concept for indoor surveying or data acquisition within the LA_SpatialUnit of the Land Administration Domain Model (LADM) is presented and proposed.
\end{abstract}

\section{INTRODUCTION}

2D cadastre has been implemented by many countries, this can be described as consisting of components such as a reference frame with geodetic network, the cadastral parcel, accurate large scale maps showing land parcels within a sector in a district, within a region in the country, a unique parcel identifier within a land information systems and a database management system to store all the information concerning land. Most developed and developing countries have a cadastral system which consists of the components as described, some well organised and others badly organised and not digital. Some of the problems associated with 2D cadastre include duplication, accessibility and flexibility of land records. Previously, land records were a collection of paper maps and written reports; these usually take several months to prepare and coupled with poor archiving systems; the retrieval of such documents were very difficult to retrieve, which brings to the fore problems associated with land acquisition, this hinders development.

Currently most cities have complex buildings and the ownership of flats in such complex structures have become difficult to represent and document. A rapid indoor survey technique is significant as this will enable the surveying of indoor spaces to be carried out in a more efficient way than extracting data from $\mathrm{CAD}$ or from other sources. The significance of representing cadastre for office or commercial lots is important for determining the value of such lots or spaces.

In this research, 3D data capturing for 3D cadastre was one of the main objectives, the authors proposed a rapid indoor building surveying methodology suitable for Malaysian 3D cadastre. A graphical user interface (GUI) was developed for $3 \mathrm{D}$ visualization of $3 \mathrm{D}$ captured data and $3 \mathrm{D}$ model reconstruction.

\section{D DATA COLLECTION AND REPRESENTATION}

In $3 \mathrm{D}$ cadastre, the methodology for data capture will depend on the $3 \mathrm{D}$ objects being collected (building, pipelines, tunnels), hence the method of data capturing may be different (Aien et al., 2011). Traditional land surveying, aerial photogrammetry and terrestrial laser scanning are the most used methods to capture the data required for cadastre.

Traditional land surveying is the oldest method for data capturing in the surveying field. Electronic Distance Measurement (EDM) is the most used device in this field. Traditional land surveying compared to photogrammetry and laser scanning method is far cheaper but not precise as laser scanning and not as fast as photogrammetry for a large area. Photogrammetry is the use of an airplane with fixed cameras to take photos of earth from a pre-planned distance. Photogrammetry can help to capture data for a large area. Laser scanning method is the newest data capturing method with a high precision result but too expensive compared to the photogrammetry and land surveying.

3D data capturing devices are introduced: the Total Station (Leica 307 TCR), laser scanner (Faro Photon 120/20) and rangefinder (LaserAce 1000). The rangefinder is used to capture indoor 3D data as shown in Figure 1. Figure 2 shows the other devices used for the data collection in this research.

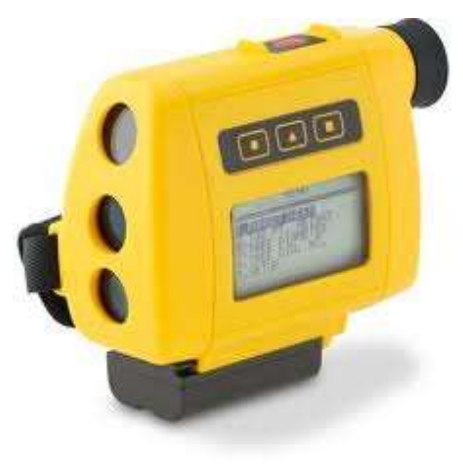

Figure 1. The rangefinder 

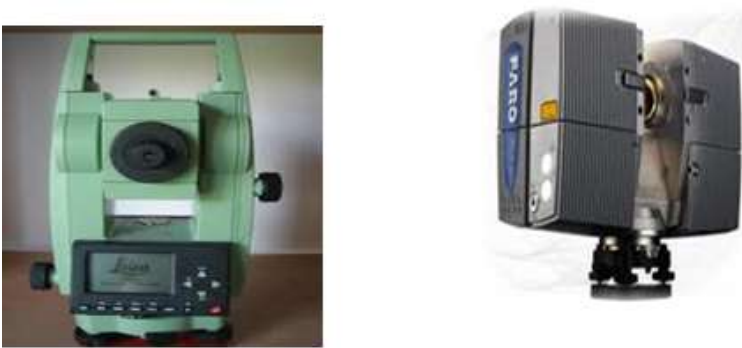

Figure 2. Leica 307 TCR (left) and Faro Photon 120/20 (right).

The rangefinder is used to survey all corners of a room and via Bluetooth technology this data is transferred into a laptop.

\section{D MODELLING}

There are many different spatial data models, some of these are categorized as: constructive solid geometry (CSG), boundary representation (B-Rep), regular decomposition, irregular decomposition and non-manifold structures (Ledoux and Gold, 2007).

For this research B-Reps and irregular decomposition models are the most relevant. B-Rep represents a solid (e.g. polyhedron) as a union of faces defined by their boundaries: edges and vertices. In this research it is assumed that faces are planar and edges are straight line segments. B-Rep models are widely used in CAD systems but they can be adapted in GIS (de Cambray, 1993).

The well-known B-Rep data structures are: half-edge (Mäntylä, 1988), winged-edge (Baumgart, 1975) and quad-edge (Guibas and Stolfi, 1985). These data structures can be used for a single 2-manifold solid representation (2-manifold is a 2D surface embedded in 3D space, e.g. a surface of a sphere, cube, or torus), but cannot be used for complex models where, for example, two or more adjacent solids have linked into one complex. A cell complex is an irregular decomposition of space or an object into non-overlapping cells of any shape. G-maps (Lienhardt, 1991) and facet-edges (Dobkin and Laszlo, 1987) are examples of data structures which can be used for construction of irregular decomposition models (e.g. for constructing a 3D Delaunay tetrahedralization).

In this paper, the dual half edge (DHE) data structure is used for 3D spatial modelling. This structure resolves some of the modelling issues in 3D GIS and expresses the geometric structures as a cell complex, in preserving adjacency relationships between cells, and including semantic information using attributes (Boguslawski, 2011; Boguslawski et al., 2011). The DHE is based on the quad-edge and its extension- the augmented quad-edge (Ledoux and Gold, 2007). These structures allow construction of models and their duals at the same time. Dual space is used to connect cells in a complex and to navigate between them. Other data structures like the halfedge or winged-edge do not provide for management of the duality.

The construction process using the DHE is based on Euler operators which are used widely in Computer Aided Design (CAD) systems for B-Rep modelling. They preserve topological consistency of the model on each step of a construction process. Euler operators were described by Baumgart (1975), Braid et al.(1980), Mäntylä (1988). 3D navigation in models is based on navigation described by Ledoux and Gold (2007) for the 3D Voronoi/Delaunay model. In this research 3D data modelling using the DHE as part of the rapid indoor data acquisition technique is addressed.

\section{LADM}

Land Administrative Domain Model (LADM) is a concept which consists of the Party, RRR's (rights, restriction, and responsibilities), Administrative, and Spatial Unit packages for a land administration system. For the purpose of this research, the focus is the Spatial Unit Package. According to Ying et al. (2011), cadastral application should have capabilities of 3D solid objects manipulation which can manage, operate and analyse 3D parcels.

In this paper, authors have decided to use DHE data structure. DHE is a topological data structure which store geometry, topology and attributes of a 3D model. Figure 3 shows prototype system for this research based on the LADM standards.

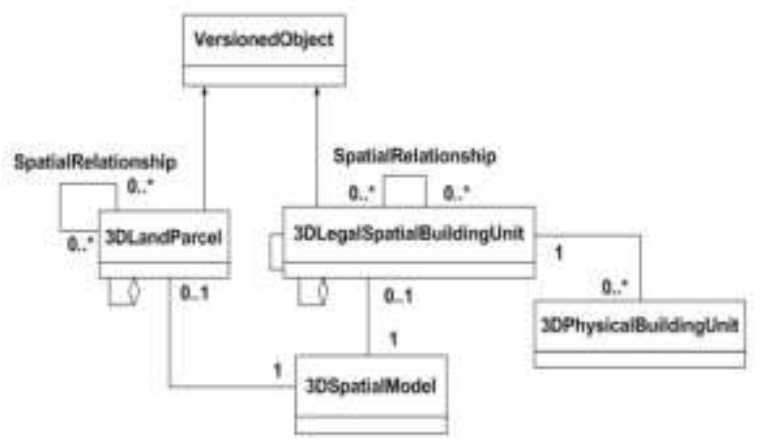

Figure 3. Prototype system based on the LADM standards

According to LADM standards ISO 19152, all classes of LADM inherit from VersionedObject. 3D parcel is stored in the 3D spatial model and similar to Ying et al. (2011) proposed prototype system, 3D land parcel and 3D legal spatial building unit are two conceptual classes for 3D space representation by 3D spatial model. In this research focus is on the 3D topological representation. Ying et al. (2011) in their paper mentioned that topological and geometrical information have to be considered together to fulfil requirements of LADM and they mentioned that two manifold solid model based on the LADM standards is insufficient for the real world 3D cadastre. They proposed their prototype system based on the Sketch up data structure which uses half-edge data structure. Boguslawski et al. (2011) in their paper described advantages of DHE over halfedge data structure for 3D modelling. (See section 3)

As can be seen in Figure 4, three classes have been designed: LADM, DHE and 3D spatial model. 3D spatial model (Figure 5 ) is modelled based on the DHE data structure and DHE is connected to the LADM class. By selecting each parcel, geometrical and topological information associated to the interested parcel can be retrieved from databases.

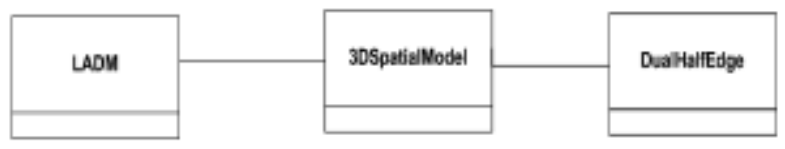

Figure 4. Relationship between LADM, DHE and 3D spatial model classes 


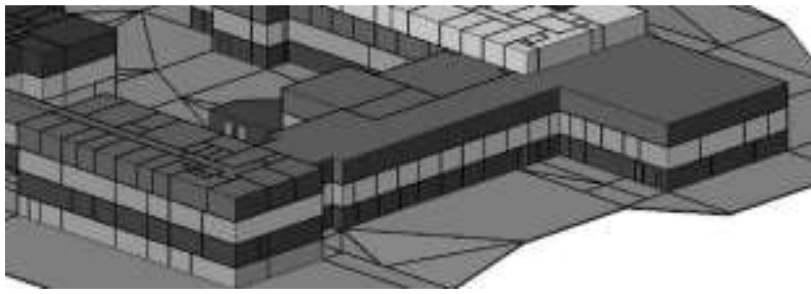

Figure 5. 3D spatial model

Each parcel is considered as a cell which has geometrical and topological information stored in the database. In the concept of DHE data structure each cell will be treated as a primal model (parcel) which is connected to the other cells (parcels) using dual model concepts. Adjacencies between rooms are considered as an important aspect of this research which is important in cadastre. Each room is considered as a cell which several attributes can be defined for each cell such as ownership number, ownership name, IC number, state and ownership type. Navigation between cells and queries like neighbourhood cells (neighbourhoods of an interested ownership) of an interested cell can be answered by dual concepts. Overlapping problem between cells (overlapping between ownerships) can be solved by defining proper tolerance for coordinates of nodes and optimizing accuracy of 3D models to an acceptable range suitable for cadastre (from preliminary results accuracy of rangefinder was \pm 10 centimetre for distance measurement and \pm 4 seconds for angle measurement). Error estimation was considered to create a precise and consistent $3 \mathrm{D}$ model suitable for 3D cadastre. Final 3D model consists of valid cells (geometry) which are connected properly (topology) modelled by DHE data structure with proper associated attributes required for cadastre (see Figure 5).

\section{PRELIMINARY RESULTS}

Captured data has been modelled to compare the results of each method. Using LaserAce 1000, 3D data capturing and 3D data modelling were simultaneously done. The rangefinder was connected to a laptop via Bluetooth; corners of rooms were captured; data was modelled on site. 3D buildings have been modelled using DHE. Time and cost were the most important factors to be considered. Figure 6 shows the 3D visualization of the $3 \mathrm{D}$ model.

a)

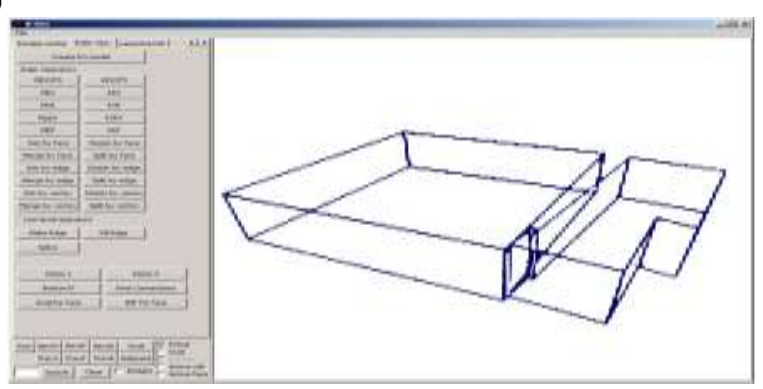

b)

\begin{tabular}{|c|c|c|c|c|c|}
\hline$x$ & t & & Used & Orignal tot: & \\
\hline 0 & 0 & 0 & TeuE & REF & \\
\hline $1-39938$ & 1278 & 11704 & TruE & \multicolumn{2}{|r|}{ 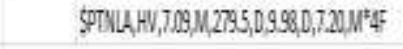 } \\
\hline $2 \cdot 25955$ & 12282 & $-7,0,6$ & TEUE & \multicolumn{2}{|r|}{ 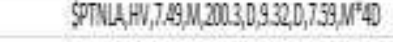 } \\
\hline 3 535888 & 11754 & -2976 & TRUE & \multicolumn{2}{|r|}{ 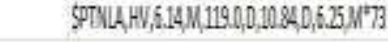 } \\
\hline 40.9354 & 12606 & 5596 & TEUE & \multicolumn{2}{|r|}{ 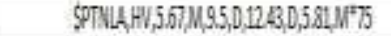 } \\
\hline
\end{tabular}

Figure 6 a) GUI for 3D data representation b) 3D model reconstruction (Boguslawski, 2011)

The results obtained from the three measuring devices were compared. Figure 7 shows three 3D models reconstructed from data collected using different devices.
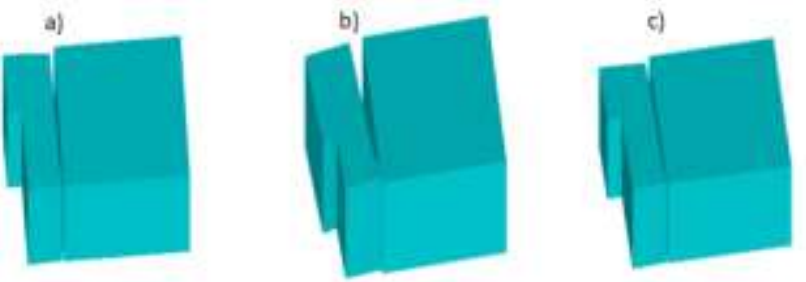

Figure 7. 3D models reconstructed from data collected by a) Leica 307 TCR, b) LaserAce 1000 and c) Faro Photon $120 / 20$

Data was collected in two rooms, processed and modelled. The surface area, cost and time for data collection were compared. Initial comparisons of these devices are shown in Figures 8- 10

LaserAce 1000 gave a higher value for the surface area as compared to the other equipment's with a difference of $2.1 \mathrm{~m}^{2}$ and $2.2 \mathrm{~m}^{2}$ between that of the Photon 120/20 and Leica 307 CR respectively (see Figure 8 ). This may be due to accurate bisection using the rangefinder.

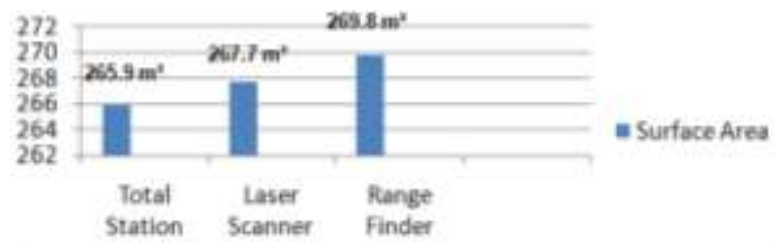

Figure 8. Equipment comparison based on model surface area

Figure 9 shows comparison of equipment in relation to setup and data collection, 10 minutes was spent for using the rangefinder, three times of that duration for the total station and fifteen times for the terrestrial laser scanner; hence the rangefinder can be used in a rapid survey. This concept can be extrapolated for complex buildings and the duration for the rangefinder will be lower.

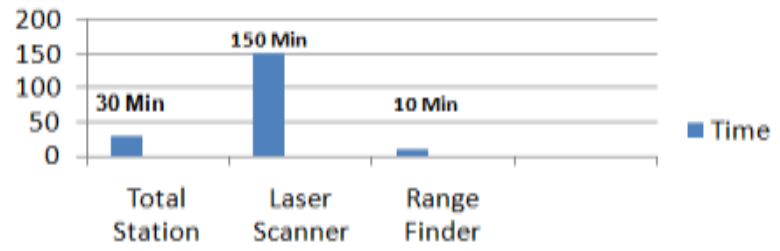

Figure 9. Equipment comparison based on the time of measurement

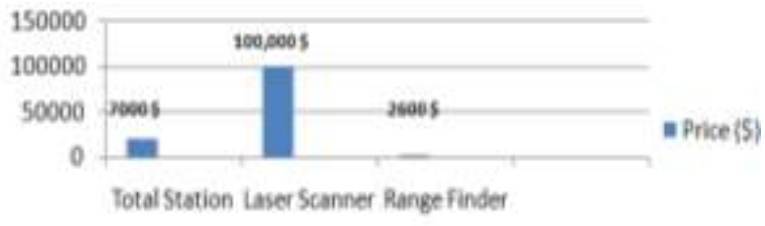

Figure 10. Equipment comparison based on the cost 
Preliminary measurements taken using the rangefinder are very promising and researchers believe that future development will improve their precision. The cost of the rangefinder is very low compared to the other devices (laser scanner and Total Station) used in this research (See Figure 10). The time necessary for data collection using the rangefinder and model reconstruction is lower than in the case of laser scanner and Total Station.

Data storage required for Faro Photon 120/20 data was 8 Giga Bytes (GB) as it was set for high resolution data and required data storage for LaserAce 1000 modelled by DHE data structure was 8 Kilo Bytes (KB). Data storage required for Total Station was $25 \mathrm{~KB}$ in CityGML data format. Data processing (preprocessing and post-processing) of Faro Photon 120/20 required high memory space and high performance workstation which significantly increase time and cost of a project.

\section{CONCLUDING REMARKS}

A rapid surveying technique for 3D indoor data acquisition has been proposed and presented. The DHE was used to model data from the rangefinder. The technique can be applied for indoor data acquisition in relation to LADM-based 3D cadastre. 3D modelling of more complex structures will be assessed in the future. Rangefinder was compared to two high accurate surveying devices (Leica 307 TCR and Faro Photon 120/20). Preliminary results of comparison between mentioned devices were promising based on the three factors: time, cost and surface area. Proposed method of data acquisition shows better result based on the cost and time factors and the modelled geometry was acceptable compared to the Leica 307 TCR and Faro Photon 120/20. Authors of this paper encountered the issue of rotated 3D models. Several tests have been conducted by the authors to solve this problem which could not be surely answered. Several possibilities for this issue can be considered:

1. Compass error

Compass problem is one of the possibilities for this issue which is factory problem and this technical problem should be solved by the Trimble Company.

2. Calibration problem

It is possible that rangefinder was not properly calibrated by the Trimble technicians which cause this rotated 3D models.

3. Room's corner material

Room corner material may cause distortion in signal reflection. Reflection of signal can be different based on the room's material.

A base station for indoor surveying is to be constructed to fully analyse the rotated 3D models problem. Nowadays, municipalities intend to have 3D city models for facility management, disaster management and architectural planning. 3D data acquisition can be done by laser scanning for indoor environment which is costly and time consuming process. Authors of this paper believe that proposed surveying technique can be employed for basic indoor environment modelling to decrease cost and time of 3D city modelling. Proposed surveying technique can be useful and affordable for most of municipalities. Proposed technique from preliminary result had accuracy of \pm 10 centimetre for distance measurement and \pm 4 seconds for angle measurement. Authors of this paper intend to investigate accuracy of LaserAce 1000 for a complex building in near future.

\section{ACKNOWLEDGMENT}

This research is supported by the Ministry of Higher Education in Malaysia (ERGS grant, vote no. 4L047, Universiti Teknologi Malaysia).

\section{References}

Aien, A, Rajabifard, A, Kalantari, M, Wiliamson, I,2011. Aspects of 3D Cadastre- A Case Study in Victoria. FIG Working Week 2011. 18-22 May. Marrakech. Morocco.

Baumgart, B., G., 1975. A polyhedron representation for computer vision, National Computer Conference and Exposition. ACM, Anaheim, California.

Boguslawski, P., Gold, C.M. and Ledoux, H., 2011. Modelling and analysing 3D buildings with a primal/dual data structure. ISPRS Journal of Photogrammetry and Remote Sensing, 66(2): 188-197.

Boguslawski, P., 2011. Modelling and Analysing 3D Building Interiors with the Dual Half-Edge Data Structure. PhD Thesis, University of Glamorgan, Pontypridd, Wales, UK, 134 pp.

Braid, I.C., Hillyard, R.C. and Stroud, I.A., 1980. Stepwise Construction of Polyhedra in Geometric Modelling. In: e. K.W.Brodlie (Editor), Mathematical Methods in Computer Graphics and Design. Academic Press, pp. 123-141.

De Cambray, B., 1993. Three-dimensional (3D) modelling in a geographical database, AutoCarto 11, Bethesda, Maryland, pp. 338-347.

Dobkin, D.P. and Laszlo, M.J., 1987. Primitives for the manipulation of three-dimensional subdivisions, Proceedings of the third annual symposium on Computational geometry. ACM, Waterloo, Ontario, Canada.

Guibas, L. and Stolfi, J., 1985. Primitives for the manipulation of general subdivisions and the computation of Voronoi Diagrams. ACM Trans. Graph., 4(2): 74-123.

ISO (TC 211), 2012, ISO 19152: Geographic information-Land Administration Domain Model (LADM).

Ledoux, H. and Gold, C.M., 2007. Simultaneous storage of primal and dual three-dimensional subdivisions. Computers, Environment and Urban Systems, 31(4): 393-408.

Lienhardt, P., 1991. Topological models for boundary representation: a comparison with n-dimensional generalized maps. Computer Aided Design, 23(1): 59-82.

Mäntylä, M., 1988. Introduction to Solid Modeling. Computer Science Press, Inc., 401 pp.

Ying, S., GUO, R., LI, L., Van oosterom,P. Ledoux,H., and Stoter,J., 2011. Design and Development of a 3D Cadastral System Prototype based on the LADM and 3D Topology, 2nd International Workshop on 3D Cadastres, 16-18 November, Delft, the Netherlands. 